\title{
Jogos educativos em dispositivos móveis como auxílio ao ensino da matemática
}

\author{
José Francisco Barbosa Neto \\ UFPE - Centro de Informática, PE - Brasil. E-mail: jose.neto86@gmail.com \\ Fernando de Souza da Fonseca \\ UFPE - Centro de Informática, PE - Brasil. E-mail: fdfd@cin.ufpe.br
}

\begin{abstract}
Resumo: Este artigo propõe a utilização de jogos educativos para dispositivos móveis como meio de estimular o aprendizado da Matemática. Para tanto, foi criado um jogo baseado na obra literária de Malba Tahan: O homem que calculava. Este jogo foi desenvolvido para dispositivos móveis com o sistema operacional Android. Visando comprovar a efetividade desta proposta, foi realizada uma avaliação segundo os critérios definidos por Medeiros \& Schimiguel (2012), a qual retratou a aceitação e a motivação das crianças de utilizar jogos educativos móveis como uma oportunidade de trabalhar conhecimentos ensinados em salas de aula tradicionais sob uma perspectiva diferente e construtivista.
\end{abstract}

Palavras chaves: Aprendizado móvel, Android, Fração matemática

Educational games on mobile devices to aid Math teaching

\begin{abstract}
This article proposes the use of educational games for mobile devices as a means of stimulating Math learning. It was created a game based on the literary work of Malba Tahan: The man who counted. This game was designed for mobile devices with the Android operating system. In order to prove the effectiveness of this proposal, an evaluation was carried out in accordance with the criteria defined by Medeiros \& Schimiguel (2012), also reported in this work. Such an evaluation highlighted children's motivation and acceptation for using mobile educational games to work out classroom's knowledge under an innovative perspective.
\end{abstract}

Keywords: m-learning, Android, Math fractions

\section{Introdução}

O uso das Tecnologias da Informação e Comunicação (TIC) aumentou as possibilidades de ampliação de acesso à formação, tornando-se parte integrante de projetos educacionais. Essas mudanças estão fornecendo novas possibilidades de práticas docentes, visando melhorar a qualidade do processo educativo (Elias 2011). 
Na última década, a difusão rápida de dispositivos móveis na sociedade, especialmente smartphones, ofereceu oportunidades de inovação no processo educacional. A aprendizagem móvel é um novo paradigma educacional, o qual é mais flexível do que a aprendizagem que utiliza computadores tradicionais. Ela permite que qualquer pessoa possa acessar informações e materiais de aprendizagem de qualquer lugar e a qualquer hora (Demirbilek 2010; Earnshaw et al. 2011) .

Segundo Calisto et al. (2010), os jogos educativos fornecem uma importante contribuição à aprendizagem, pois são ambientes capazes de disseminar informações. De fato, um jogo educativo estimulará a atenção dos alunos, ao mesmo tempo em que os motivará através de atividades de competição e cooperação.

A prática docente que utiliza o jogo como uma ferramenta de apoio ao processo de aprendizagem, além de permitir a integração de diferentes áreas do conhecimento, nas quais conteúdos se encontram transversalmente nos domínios da educação ambiental, biologia, física, química, matemática, informática, entre outros, oferece algumas vantagens como ludicidade, cooperação, participação, prazer e motivação (Souza et. al 2010).

Este trabalho tem como objetivo apresentar um estudo da viabilidade da utilização de jogos educativos digitais desenvolvidos para dispositivos móveis no auxílio do ensino da matemática. Ele está organizado em 6 (seis) seções: além desta, a seção 2 (dois) apresenta uma visão geral de jogos digitais na educação; a seção 3 (três) apresenta conceitos básicos sobre mobile leraning; a seção 4 (quatro) apresenta o jogo desenvolvido; a seção 5 (cinco) apresenta a avaliação realizada e os resultados obtidos e a seção 6 (seis) apresenta as considerações finais, e é seguida pelas referências bibliográficas utilizadas.

\section{Jogos Educativos Digitais}

O crescimento da indústria de jogos tem despertado interesse entre os pesquisadores de tecnologia educacional. A aprendizagem através de jogos é considerada uma atividade importante, especialmente no início da infância. Particularmente, jogar é considerado uma importante atividade para o desenvolvimento psicológico, social e cognitivo. Outra vantagem da utilização de jogos é que se pode estimular a motivação interna dos jogadores pelo desafio (De Freitas \& Mahag 2011).

Segundo Gros (2007), aprendizagem é vista por muitas pessoas como um "dever" para as crianças. Esta visão crítica faz com que a aprendizagem seja encarada como um trabalho. Entretanto, quando uma atividade envolve prazer, diversão, motivação, interesse e paixão, o indivíduo é capaz de dedicar a ela uma grande parte do tempo e esforço. Como jogos digitais envolvem muitos fatores motivacionais, poderiam ser estimulados por educadores para auxiliar na aprendizagem de conteúdos difíceis de tratar em salas de aula tradicionais.

A principal ideia é que os jogos digitais, como ferramentas educacionais, podem ajudar para o desenvolvimento de conhecimento e habilidades cognitivas, como a resolução de problemas, o pensamento estratégico, a tomada de decisão, entre outras, propiciando uma compreensão mais profunda de certos princípios fundamentais de determinados assuntos (Brom et al. 2011). 
Entre os benefícios, a interatividade estabelece um ciclo de feedback muito curto com o jogador, já que os jogos podem acompanhar as ações dos alunos para determinar se as metas de aprendizagem estão a ser alcançadas. Além disso, os jogos podem utilizar a informação recolhida a partir da interação do usuário e conduzir a adaptação on-line da experiência educacional (Del Blanco et al. 2009).

Jogos aumentam as possibilidades dos alunos obterem informações, combinando com a diversão. Funcionam como uma atividade complementar de aprendizagem, servindo como instrumento de introdução a novos conhecimentos, motivação do aprendiz ou de fixação de conhecimento. Por outro lado, a popularização de jogos desenvolvidos para dispositivos móveis aponta ser essa uma boa alternativa para inclusão de informações pedagógicas em formato de mídia digital para o aprendizado (Brom 2011; Elias 2011).

\section{Mobile Learning}

Kukulska-Hume \& Traxler (2005), definem mobile learning(m-learning) como um conceito novo que envolve a mobilidade e o estudante, o qual pode realizar atividades educacionais sem a limitação do espaço físico de um ambiente de ensino. Pode-se aprender em ambientes fora das salas de aula, basta apenas motivação de aprender e um dispositivo móvel, leve e que caiba no bolso, como um celular ou um tablet, por exemplo. Como benefícios da m-learning podem ser apontados:

- Melhorar os recursos para o aprendizado do aluno, o qual os utilizará importantes para execução de tarefas, como por exemplo, consulta de informações via Internet, utilização da câmera digital, gravação de sons, troca de mensagens e outras funcionalidades existentes;

- Acessar conteúdos didáticos em qualquer lugar e a qualquer momento, de acordo com a conectividade do dispositivo;

- Aumentar a interatividade com professores e as estratégias de aprendizado disponíveis, através de novas tecnologias que dão suporte tanto à aprendizagem formal quanto à informal; e

- Fornecer meios para o desenvolvimento de métodos inovadores de ensino e de treinamento, utilizando os novos recursos de computação e de mobilidade.

A tecnologia funciona como um complemento na educação. Explorando este conceito, os dispositivos móveis tornam a educação portável de um ambiente para outro, como da sala de aula para a casa do estudante. Assim, permite que o potencial da aprendizagem seja contínuo. No entanto, aprendizagem móvel não se destina a substituir o aprendizado em sala de aula, mas pode ser visto, como um acessório que aumenta as possibilidades e estímulos para a aprendizagem (Earnshaw et al. 2011). Kukulska-Hume \& Traxler (2005) defendem que aprendizagem móvel não aponta para um tipo diferente de aprendizagem, mas uma aprendizagem comum em condições e ambientes diferentes.

O aumento do uso social dos jogos de entretenimento em dispositivos móveis, aliado com as implantações de novas técnicas de imersão no ensino-aprendizagem corrobora a premissa de que o aprendizado baseado em jogos educativos móveis é uma 
abordagem válida para apoiar abordagens formais de aprendizagem (De Freitas \& Mahag 2011).

\section{Ensino da matemática utilizando um jogo digital para dispositivos móveis}

Existem alguns conteúdos que professores enfrentam algumas dificuldades de ensinar. Dentre eles, a Matemática ocupa um lugar de destaque. Estudos como os de Mayo (2007) e Del Blanco et al. (2009) mostram que jogos especialmente sobre Matemática e Física, ajudam a melhorar o progresso de aprendizagem. Segundo Fiorentini \& Lorenzato (2006), no Brasil, fração matemática é um exemplo de um conteúdo que os alunos têm bastante dificuldade de aprender. Por tais razões, o domínio escolhido a ser trabalhado foi conhecimentos básicos da Matemática.

Assim, foi desenvolvido um jogo educativo com objetivo de apresentar de uma forma diferente, situações e problemas que a criança precisasse mobilizar conhecimentos desenvolvidos em sala de aula. Observando-se a extensa literatura brasileira, o enredo do jogo foi baseado no livro: O homem que calculava, um clássico brasileiro traduzido para o inglês e o espanhol, do escritor Malba Tahan, pseudônimo do professor de Matemática Júlio Cesar de Mello e Souza. Esse livro mantém o valor pedagógico comum em todas as obras de Malba Tahan que contam as proezas matemáticas do calculista persa Beremiz Samir, as quais se tornaram lendárias na antiga Arábia, por relatar soluções fantásticas para problemas aparentemente insolúveis vividas pelo personagem principal.

O referido jogo foi desenvolvido seguindo o processo de desenvolvimento definido por Barbosa Neto (2012), o qual é dividido em 04 atividades: Análise e Planejamento, Modelagem do Jogo, Implementação e Testes com especialistas. Em razão da plataforma Android (Android 2013) possuir código aberto, sua facilidade de distribuição e vir atraindo grande número de desenvolvedores e empresas interessadas no seu uso em dispositivos móveis como smartphones e tablets, ela foi escolhida como plataforma para o desenvolvimento deste trabalho.

Para instalar o jogo basta um dispositivo móvel que tenha o sistema operacional Android com versão a partir da 2.3.3.

O jogo foi dividido em seis estágios, para se tornar mais atrativo e motivador, com níveis de dificuldade crescente.

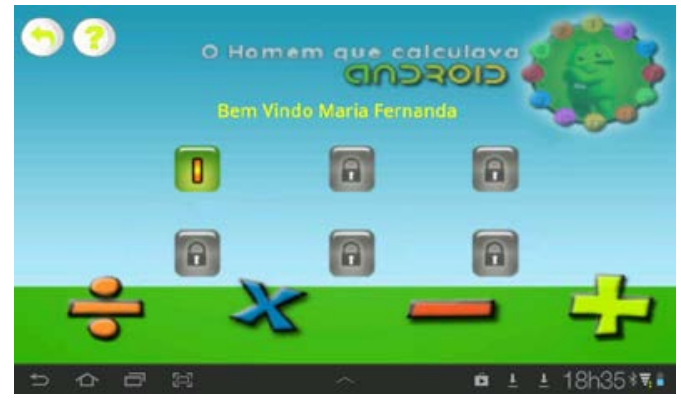

Figura 1 - Menu de estágios 
Os estágios disponíveis para jogar estarão com uma imagem contendo os números de cada um visível, enquanto que os indisponíveis estarão com uma imagem de um cadeado, como apresentado na Figura 1. Ao começar o jogo, apenas o primeiro estágio estará disponível, pois, a disponibilidade de um estágio depende da conclusão com sucesso do seu antecessor.

No primeiro estágio, o aluno reproduz a aventura vivida por Beremiz, personagem principal. Neste estágio, Beremiz está viajando com um amigo pelo deserto, montados em um único camelo. Durante a viagem eles encontram três irmãos discutindo como irão dividir uma herança deixada de 35 camelos de acordo com a regra estabelecida pelo pai. Esta regra define que o filho mais velho receberá metade da herança, o segundo filho receberá um terço da herança e o filho mais novo receberá um nono da herança. Ao iniciar qualquer estágio, o jogo fornece uma tela de instruções do estágio, apresentada na Figura 2.

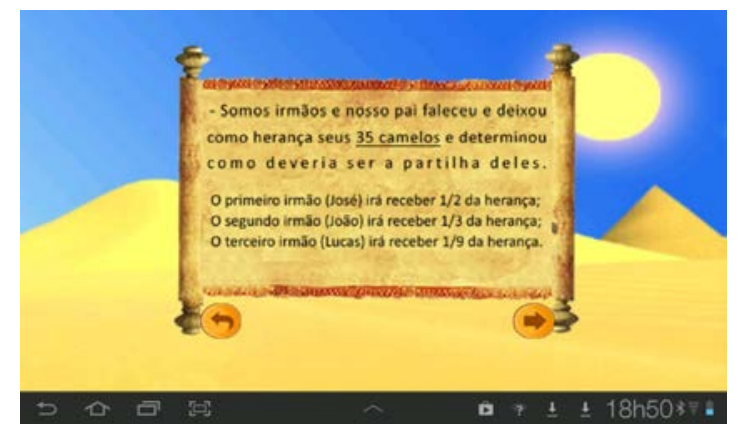

Figura 2- Tela de instruções

Após o aluno ler as instruções, ele terá que fazer a divisão dos camelos. Neste momento o jogo apresentará uma tela (Figura 3), na qual ele tentará dividir os 35 camelos para os três irmãos.

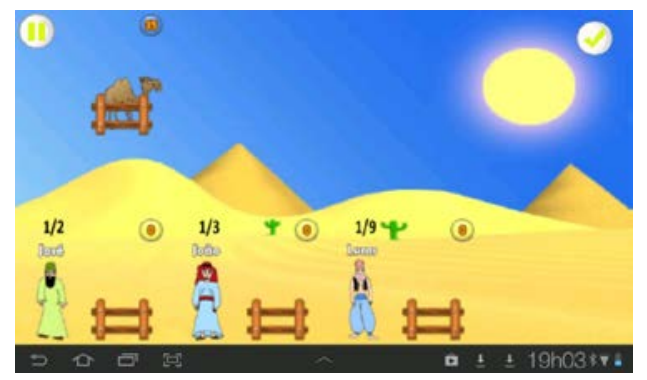

Figura 3 - Divisão dos 35 camelos

O problema é que com os 35 camelos a divisão entre os irmãos fica inexata, não sendo possível a qualquer um deles receber apenas parte de um camelo. Então, o aluno deverá perceber que não existe uma solução satisfatória para todos, e por este motivo os irmãos foram encontrados brigando. Após ele tentar encontrar uma solução para o caso, o jogo irá apresentá-lo uma nova tela de instruções, mostrando que o calculista doou seu camelo para os irmãos como parte da herança e com isto resolveu o problema da inexatidão do cálculo, e irá receber a sobra dos camelos como pagamento pela ajuda. Então, nesse momento o estudante precisará fazer uma nova divisão, agora contemplando os viajantes, assim, totalizando 36 camelos (Figura 4) 


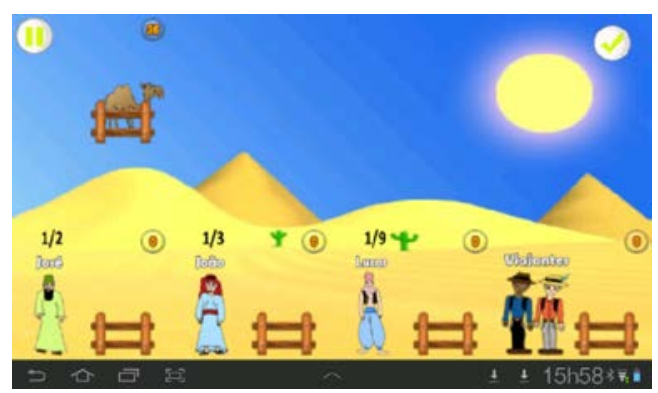

Figura 4 - Divisão dos 36 camelos

Após realizar a divisão, deverá clicar o botão de concluir (canto superior direito da tela). Se a divisão estiver incorreta, o jogo exibirá uma mensagem informando que a resposta está incorreta e o estágio recomeçará. Caso a divisão esteja correta, o sistema exibirá uma mensagem indicando o sucesso na divisão e encaminhará para tela de conceito (Figura 5).

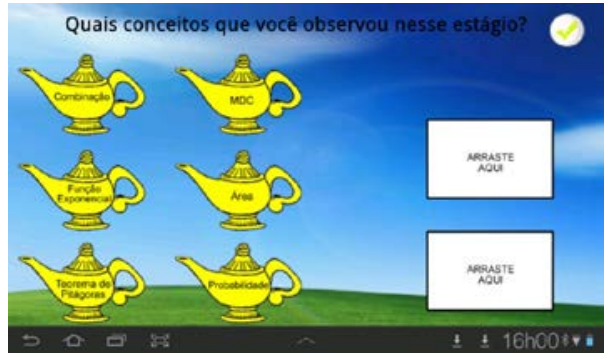

Figura 5 - Tela de conceito

Nesta tela o aluno irá selecionar os conceitos matemáticos (conteúdos) aprendidos em sala de aula, dos quais conseguiu perceber a utilização para resolução do problema anterior. Neste estágio o aluno deverá indicar que percebeu a utilização de fração e adição para resolver o problema. Uma tela semelhante a esta estará presente após a conclusão de todos os estágios do jogo.

\section{Avaliação e Resultados Obtidos}

Silva (2009) afirma que o professor ao escolher um software educacional adequado, precisa ter como critérios: o conceito a ser ensinado, o conhecimento sobre a usabilidade com os alunos, o seu manuseio pessoal, para, após, avaliar e planejar uma situação de ensino. A avaliação de software educacional deve incluir aspectos como usabilidade, a eficácia das ferramentas de auxílio, além da aprendizagem alcançada. Elas enfatizam a necessidade de utilizar tanto a avaliação orientada para o produto quanto a avaliação orientada para o usuário.

A avaliação deste jogo foi realizada segundo os critérios de Medeiros \& Schimiguel (2012) :

- Qualidade do conteúdo - Veracidade e apresentação equilibrada das ideias com nível apropriado de detalhes, enfatizando os pontos significantes; 
- Alinhamento do Objetivo da Aprendizagem - Alinhamento entre as metas de aprendizagem, atividades, avaliações e características dos alunos;

- Motivação - Capacidade de motivar o interesse do jogador;

- Imersão - Capacidade de envolver o jogador profundamente;

- Objetivos Claros - Metas claras do que deve ser realizado;

- Feedback e Adaptação - Elaboração do feedback positivo e negativo do jogo. Conteúdo que se adapta de acordo com a habilidade do jogador;

- Apresentação - Informação visual;

- Interação Social - Dispor meios de interação com outros jogadores; e

- Reusabilidade - Capacidade de ser utilizado em diferentes contextos de aprendizagem e com alunos de diferentes idades e interesses.

Godoi \& Padovani (2011) observaram que instrumentos avaliativos de software educacional podem ser classificados em: checklists, diretrizes, escalas de avaliação, formulários, modelo conceitual, questionários e sistemas ou de forma híbrida. Dentre os instrumentos avaliativos, foi escolhido o questionário de avaliação para verificar o impacto do jogo no processo de ensino-aprendizagem da matemática.

\subsection{Resultados Obtidos}

Os participantes do experimento realizado com o jogo foram 16 (dezesseis) alunos oriundos de duas escolas do Recife, sendo 04 (quatro) alunos de uma escola particular (cursando o $8^{\circ}$ ano do ensino fundamental) e 12 alunos de uma escola pública estadual (cursando o $1^{\circ}$ ano do ensino médio). Neste estudo foram colocados os alunos para usarem o jogo livremente com um tempo máximo de duração de 2 horas. Cada aluno portava um dispositivo móvel, os quais foram estes utilizados: Tablet Samsung Galaxy Tab 2 7” (Android 3.0), Tablet DHL 7” (Android 2.3.3), Smartphone Samsung Galaxy SII (Android 4.0.1). A avaliação realizada buscou responder alguns questionamentos, tais como:

- O aluno conseguiu utilizar o sistema e compreender suas funções com pouco esforço?

- O aluno conseguiu perceber os conteúdos matemáticos trabalhados em sala de aula sob a perspectiva de um jogo?

- O aluno sentiu-se motivado durante o jogo?

- A imersão com o jogo foi satisfatória?

- O aluno ficou motivado e aprovou a utilização de jogos educativos como complemento de sala de aula?

- Explorar a mobilidade foi interessante para os alunos?

Para conseguir respostas aos questionamentos, ao final do jogo, foi aplicado um questionário individualmente para cada aluno. Para a análise dos resultados foi utilizado o método de frequência relativa em todas as questões do questionário aplicado aos participantes. Tal questionário apresentava perguntas assertivas e o aluno tinha que escolher as opções de: Discordo Totalmente (DT), Discordo Parcialmente (DP), 
Indiferente (IN), Concordo Parcialmente (CP) e Concordo Totalmente (CT). As principais questões sãoapresentadas na Tabela 1.

Tabela 1 - Principais resultados da avaliação

\begin{tabular}{|l|c|c|c|c|c|}
\hline \multicolumn{1}{|c}{ Pergunta } & DT & DP & IN & CP & CT \\
\hline $\begin{array}{l}\text { Entendi rapidamente o que deveria fazer para } \\
\text { interagir com o jogo. }\end{array}$ & $0 \%$ & $0 \%$ & $12 \%$ & $19 \%$ & $69 \%$ \\
\hline $\begin{array}{l}\text { O jogo reconheceu corretamente meus } \\
\text { movimentos (toques) }\end{array}$ & $0 \%$ & $0 \%$ & $0 \%$ & $6 \%$ & $94 \%$ \\
\hline As imagens estavam adequadas & $0 \%$ & $0 \%$ & $0 \%$ & $6 \%$ & $94 \%$ \\
\hline Compreendi facilmente as instruções & $6 \%$ & $0 \%$ & $6 \%$ & $19 \%$ & $69 \%$ \\
\hline $\begin{array}{l}\text { Consegui identificar assuntos dados em sala } \\
\text { de aula }\end{array}$ & $0 \%$ & $7 \%$ & $6 \%$ & $31 \%$ & $56 \%$ \\
\hline Achei que faltaram explicações nas instruções & $75 \%$ & $12 \%$ & $13 \%$ & $0 \%$ & $0 \%$ \\
\hline $\begin{array}{l}\text { Senti-me motivado para avançar pelos } \\
\text { estágios }\end{array}$ & $14 \%$ & $0 \%$ & $13 \%$ & $0 \%$ & $73 \%$ \\
\hline O objetivo do jogo não estava claro & $81 \%$ & $19 \%$ & $0 \%$ & $0 \%$ & $0 \%$ \\
\hline $\begin{array}{l}\text { A mobilidade ajuda bastante, pois, não } \\
\text { preciso estar em um lugar determinado para } \\
\text { jogar }\end{array}$ & $0 \%$ & $0 \%$ & $6 \%$ & $13 \%$ & $81 \%$ \\
\hline O jogo foi atrativo e interessante & $0 \%$ & $0 \%$ & $0 \%$ & $6 \%$ & $94 \%$ \\
\hline $\begin{array}{l}\text { Instalaria o jogo no meu dispositivo móvel e } \\
\text { recomendaria para meus amigos usarem }\end{array}$ & $0 \%$ & $0 \%$ & $12 \%$ & $13 \%$ & $75 \%$ \\
\hline
\end{tabular}

Os resultados obtidos pela aplicação dos questionários apontam que a maioria dos participantes respodeu de forma positiva sobre a usabilidade do jogo. Pois, $100 \%$ dos participantes concordaram que o jogo reconheceu corretamente seus movimentos (toques), enquanto outros 88\% concordaram com a afirmativa: "entendi rapidamente o que deveria fazer para interagir com o jogo”, apontou a satisfação ao interagir com a aplicação.

A maioria dos alunos (87\%) concordou que conseguiram identificar os conteúdos trabalhados em sala de aula pelos professores no jogo. Este resultado é importante, pois reflete que o aluno conseguiu enteder os conteúdos trabalhados na sala de aula e usá-los sob uma perspectiva diferente. Outro resultado que desperta atenção é a motivação dos alunos, já que todos concordaram que se sentiram motivados à medida que foram avançando pelos estágios.

Os resultados também apontaram que a maioria dos alunos aprovou o apoio da mobilidade na utilização de jogos educativos digitais, por facilitar a utilização, pois não precisava estar fixo a um lugar específico para poder utilizar o jogo. Por último e não menos importante, $88 \%$ dos alunos concordaram que instalaria esse jogo em seu dispositivo e recomendariam para seus amigos utilizarem.

Um mês após a realização do experimento com os alunos, foi apresentado um questionário para os professores de matemática dos alunos participantes. O resultado 
encontradro foi que $86 \%$ dos participantes estavam mais motivados com o processo de ensino-aprendizagem e participativos durante as aulas. Apesar de ter sido detectado pelos professores uma melhoria de rendimento escolar dos participantes, não foi possível comprovar cientificamente, com os experimentos realizados, que tal melhoria se deu em virtude da utilização de jogos educativos

\section{Considerações Finais}

Atualmente, crianças começam muito cedo a utilizar computadores e o uso de jogos é uma prática bastante comum presente nessa etapa. Entretanto, a utilização de jogos educativos em ambientes escolares ainda é pouco explorada como forma de fixação e auxílio do aprendizado no Brasil.

A mobilidade é um fator que pode ser explorado pela educação, pois, dispositivos móveis tornaram-se uma peça fundamental no cotidiano das pessoas nestes últimos anos. Esse tipo de dispositivo permite realizar as atividades no tempo que for mais conviniente ao aluno, sua à principal vantagem. $\mathrm{O}$ aluno pode, por exemplo, aproveitar ocasiões como deslocamentos e filas de espera para atendimento para realizar atividades educacionais em seus dispositivos móveis.

A principal contribuição deste trabalho foi utilizar um recurso de TI por meio de um jogo digital móvel para auxiliar os alunos no aprendizado de matemática. Com esta abordagem construtivista ele trabalha conceitos aprendidos em sala de aula sob uma perspectiva diferente, uma vez que ele está acostumado com problemas rotineiros apresentados pelo professor em sala de aula. Abordagens que fogem do comum acabam despertando maiores interesses nos alunos.

Isto permite que a utilização de todos os benefícios da mobilidade para atrair a atenção de jovens, os quais atualmente estão cada vez mais adeptos da tecnologia. Esta possiblidade de utilizar jogos aliados com a mobilidade despertou bastante interesse nos jovens alunos, conforme comprovada pela avaliação realizada.

Outra contribuição foi conseguir transformar um enredo de um livro de sucesso da literatura brasileira, admirado por milhares de estudantes e professores, em um divertido jogo educativo, permitindo ao ato de jogar transformar-se em uma atividade pedagógica. O jogo está sendo adptados a diversos tipos de dispositivos móveis para ser publicado na Google Play Store (Google Play, 2013) para ser distribuído gratuitamente.

\section{Referências Bibliográficas}

ANDROID disponível em: http://developer.android.com/index.html último acesso: 05/04/2013

BARBOSA NETO, J. F. Uma metodologia para desenvolvimento de jogos educativos em dispositivos móveis para ambientes virtuais de aprendizagem. Recife: UFPE, 2012. 136p. Dissertação de Mestrado 
BROM, C., PREUSS, M. e KLEMENT, D. Are educational computer micro-games engaging and effective for knowledge acquisition at high-schools? A quasiexperimental study. Computers \& Education, 57(3), p.1971-1988. 2011

CALISTO, A.; BARBOSA D.; SILVA C. Uma Análise Comparativa entre Jogos Educativos Visando a Criação de um Jogo para Educação Ambiental. In: XXI Simpósio Brasileiro de Informática na Educação, João Pessoa, PB, 2010.

DE FREITAS, S.; MAHARG, P. Digital Games and Learning. Continuum International Publishing Group. 2011.

DEL BLANCO, Á.; TORRENTE, J.; MORENO-GER, P.; FERNÁNDEZ-MANJÓN, B. A General Architecture for the Integration of Educational Videogames in Standards-compliant Virtual Learning Environments. In: Ninth IEEE International Conference on Advanced Learning Technologies ICALT, p.53-55. 2009

DEMIRBILEK, M. Investigating attitudes of adult educators towards educational mobile media and games in eight European countries. Journal of Information Technology Education, v9, p 235-247, 2010.

ELIAS, T. Principles for Mobile Learning. In: International Review of Research in Open and Distance Learning, 12(2), p.143-156. 2011.

EARNSHAW, R. A.; ROBISON, D.; EXCELL, P. S. From E-Learning to M-Learning the use of Mixed Reality Games as a new Educational Paradigm. International Journal of Interactive Mobile Technologies iJIM v5, p17-25, 2011.

FIORENTINI, D.; LORENZATO, S. Investigação em Educação Matemática: percursos teóricos e metodológicos. Autores Associados, 2ª edição, 2006.

GODOI, K.; PADOVANI, S., Instrumentos avaliativos de software educativo: uma investigação de sua utilização por professores. Estudos em Design, v. 19, p. 1-23, 2011.

GOOGLE PLAY disponível em: http://play.google.com último acesso: 05/04/2013

GROS, B. The Design of Learning Environments Using Videogames in Formal Education. In: First IEEE International Workshop on Digital Game and Intelligent Toy Enhanced Learning DIGITEL07. IEEE, pp. 19-24, 2007.

KUKULSKA-HULME, A.; TRAXLER, J. Mobile Learning: A handbook for educators and trainers. Routledge, 2005.

MAYO, M. J. Special issue on Creating a Science of Games. Communications of the ACM, v50(7) p.26-73. 2007.

MEDEIROS, M. de O; SCHIMIGUEL, J.; Uma abordagem para avaliação de jogos educativos: ênfase no ensino fundamental. RENOTE - Revista de novas tecnologias na educação, v 10, n3, 2012.

SILVA, R. J. S. Avaliação de Software Educacional: critérios para definição da qualidade do produto. In: Simposio Nacional ABCiber, v. 1, p. 1-15. 2009.

SOUZA, M. M.; RESENDE R. F.; PRADO L. S.; FONSECA, E. F.; CARVALHO, F. A.; RODRIGUES, A. D. SPARSE: Um Ambiente de Ensino e Aprendizado de Engenharia de Software Baseado em Jogos e Simulação. In: XXI Simpósio Brasileiro de Informática na Educação, João Pessoa, PB, 2010 